\title{
Arbeitsfähigkeit und Invalidenversicherung
}

\author{
R. Conne
}

Bei der Diskussion um den Missbrauch der Invalidenversicherung und um «Scheininvalide» ist Polemik nicht angebracht, aber es bestehen mannigfaltige Probleme. Die Ärzte sind nur ein Teil des Systems und können nicht alle Probleme lösen. Alle Beteiligten wie Unternehmer, Politik, Versicherungen, Rechtsanwälte und Gerichte sind gefordert. Risikopatienten müssen frühzeitig erfasst und durch ein aktives Case-Management unterstützt werden. Arbeitsplätze für vermindert belastbare Personen sind bereitzustellen. Für den Wiedereinstieg ins Erwerbsleben müssen Anreize geschaffen werden. Die Prozesse der Abklärung und die Versicherungsabläufe sind zu professionalisieren und zu beschleunigen, und die Rechtsprechung ist zu überdenken.

1 CHSS 2003 und BSV IV-Statistik 2003

2 Sendung des SF1 in 10 vor 10 (Ende August/Anfang September)

3 Meine J. Die ärztliche Unfallbegutachtung in der Schweiz erfüllt sie die heutigen Qualitätsanforderungen? Swiss Surgery 1998;4(2):53-7.

4 Murer E. Die UV- und IV-rechtliche Auseinandersetzung mit reaktiven psychischen Störungen: Eine Zwischenbilanz. In: Psychische Störungen und die Sozialversicherung - Schwerpunkt Unfallversicherung. Bern: Verlag Stämpfli; 2002

5 Breitenmoser B, et al. «Eingliederung vor Rente» - realisierbares Ziel oder bloss wohl tönender Slogan? CH SS 1999

6 Weltwoche Nr. 17.03

7 Wird noch publiziert.

\section{Korrespondenz:}

Dr. med. Rudolf Conne

Psychiatrie und Psychotherapie FMH Beethovenstrasse 3

8002 Zürich

E-Mail: rudolf-conne@bluewin.ch
Die aktuelle Diskussion um den Missbrauch der Invalidenversicherung (IV) und um «Scheininvalide» suggeriert ein Malaise. Das führt zur

\section{Frage: \\ Handelt es sich nur um Wahlkampf- getöse oder um ein echtes Problem?}

In den letzten zwei Jahrzehnten haben sich die Rentenleistungen der IV rund vervierfacht [1], entsprechend sind auch die UVG- und die BVGRenten gestiegen. Das ist schon alarmierend. Dass in anderen Ländern eine ähnliche Entwicklung stattfindet, ist kein Trost. Bei dieser enormen Zunahme der Invaliditäten spielt der Anteil der psychisch Bedingten eine grosse Rolle. Die Zunahme kann zum Teil durch kaum beeinflussbare Faktoren erklärt werden. Z. B. die Rezession: Menschen mit psychischer Behinderung haben während der Hochkonjunktur Nischenarbeitsplätze gefunden. In der Rezession fallen sie aus dem Arbeitsprozess heraus, weil sie den stets steigenden Anforderungen im Berufsleben nicht mehr gewachsen sind. Durch die Migration haben wir einen Zuwachs an Menschen mit belastenden Erfahrungen, oft geringer Ausbildung und mit Entwurzelungsproblemen, die oft auch überlastet sind (Frauen mit Haushalt, Kindern und voller Berufstätigkeit). Eine wichtige Rolle spielt aber auch das vermehrte Erfassen von psychischen Störungen. Die psychiatrische Versorgung war lange Zeit unterentwickelt. Als ich vor 24 Jahren in Winterthur eine Praxis eröffnete, gab es in Frauenfeld keinen einzigen Psychiater (weder einen freipraktizierenden noch den externen psychiatrischen Dienst von Münsterlingen). Die Gleichstellung von psychischen Störungen mit somatischen ist noch jüngeren Datums. Eine weitere Zunahme von IV-Fällen ist zu erwarten. Bei den Sozialämtern sind noch Menschen hängig, welche Anspruch haben auf Leistungen der IV [2].

Es stellt sich nun die Frage, ob die erwähnten Faktoren genügen, um die Zunahme der Berentungen in vollem Ausmass zu erklären. Es gibt warnende Stimmen. So hat Meine [3] festgestellt, dass die Qualität der versicherungsmedizinischen Gutachten in der Schweiz überwiegend ungenügend sei. Wenn man bedenkt, dass die wohl wichtigste Aussage eines versicherungsmedizinischen Gutachtens diejenige zur Arbeitsfähigkeit ist, macht dies nachdenklich. Murer [4] hat am Freiburger Sozialrechtstag 2002 gesagt, die Ärzte würden schreiben, was ihre Patienten wünschen, damit sie diese nicht verlieren. Breitenmoser [5] schreibt, es gebe dort eine hohe Rate von Invaliden, wo viele Ärzte/Psychiater praktizierten. Zwischen den Zeilen sagt sie, die Ärzte würden die Arbeitsfähigkeit unkritisch beurteilen. Besonders alarmierend sei die $\mathrm{Zu}-$ nahme von jungen IV-Rentnern. In der Weltwoche [6] erschien vor kurzem ein Artikel «Ein Volk von IV-Rentnern». Im Juni 2003 fand in Luzern ein Kongress statt, organisiert von der Universität St. Gallen, zum Thema Schmerz und Arbeitsunfähigkeit [7]. Dort wurde erwähnt, dass in den versicherungsmedizinischen Gutachten häufig ein Sprung besteht zwischen der Diagnose und der Arbeitsunfähigkeit. Es wurde von seiten der Juristen gewünscht, dass eine ausführliche Begründung für die Einschätzung der Arbeitsunfähigkeit/Arbeitsfähigkeit gegeben werde. Diese soll sich auf mehr Fremdangaben stützen und die Einschätzung plausibel machen. Meine eigene Erfahrung ist die, dass die Beurteilung der Arbeitsunfähigkeit/Arbeitsfähigkeit schwierig ist. Das sehen wir immer wieder in unserem Qualitätszirkel. Ausserdem habe ich einen Fall erlebt, der zur gleichen Zeit von einem Psychiater in Tübingen und einem Psychiater in Zürich begutachtet wurde. Der Psychiater in Tübingen kam auf eine 20prozentige Arbeitsunfähigkeit, der Zürcher Psychiater auf eine 100prozentige.

Die Antwort auf die erste Frage könnte etwa so lauten: Es besteht ein echtes Problem. Allerdings ist Polemik nicht gerechtfertigt. Missbrauch ist in der Invalidenversicherung selten. Hingegen existieren mannigfaltige Schwierigkeiten. Dies führt zur 


\section{Frage: \\ Worin bestehen die Schwierigkeiten bei der Beurteilung der Arbeitsfähigkeit?}

Wir Ärzte haben keinerlei Ausbildung im Beurteilen der Arbeitsfähigkeit erhalten. Wenn ein Patient mit $40^{\circ}$ Fieber im Bett liegt, ist diese Beurteilung auch nicht schwierig. Aber bei einer längeren Arbeitsunfähigkeit, z. B. bei Depression oder Rückenschmerzen, stellt sich die Frage, was der Patient noch kann und was ihm noch zumutbar ist.

Es gibt einen wissenschaftstheoretischen Aspekt dieser Frage. Die Medizin ist die Lehre von den Krankheiten bzw. Gesundheitsstörungen und deren Behandlung. Wir sind wissenschaftsgläubig. Was die Gesellschaft von uns verlangt, nämlich die Beurteilung der Arbeitsfähigkeit, kann nicht durch eine wissenschaftliche Methode beantwortet werden. Es besteht ein wichtiger Unterschied zwischen Medizin als Wissenschaft - besser Wissenschaften - und Medizin als Praxis. Ich gebe ein Beispiel: Es kommt ein Patient mit Depression in die Praxis. Wenn ich seine Depression als Folge von inneren Konflikten oder äusseren psychosozialen Belastungen verstehe, behandle ich ihn mit Psychotherapie. Psychotherapie ist ein medizinisches Denksystem, eine Wissenschaft (genauer: es gibt mehrere verschiedene Psychotherapieschulen und somit mehrere psychotherapeutischen Wissenschaften). Verstehe ich aber die Depression meines Patienten als Ausdruck eines Mangels an Neurotransmittoren, so behandle ich ihn mit antidepressiven Medikamenten. Die biologische Psychiatrie ist eine andere Wissenschaft. Was zur Anwendung gelangt, ist eine Angelegenheit der Praxis und hängt von vielem ab, wie z. B. von der Art der Depression, von der Einschätzung und Grundhaltung des Arztes, von Vorlieben und der Ansprechbarkeit des Patienten. Wissenschaft ist immer ein Teilaspekt. Die Praxis hat viel mit gesundem Menschenverstand zu tun und ist die Integration verschiedener wissenschaftlicher Teilaspekte unter praktischen Gesichtspunkten. Die Arbeitsfähigkeit hat körperliche, psychische und soziale Aspekte und ist somit eine Angelegenheit der medizinischen Praxis.

Ein weiteres Element ist folgendes: Die Medizin ist traditionell eher auf das Feststellen von Defekten ausgerichtet. In diesem Zusammenhang ist zu beachten, dass ich im Titel «Arbeitsfähigkeit» habe und nicht «Arbeitsunfähigkeit». Bei der Arbeitsfähigkeit sind nicht nur die Defizite wichtig, sondern das Erfassen der Ressourcen eines Patienten. Dies ist uns viel weniger vertraut. Um dies beurteilen zu können, ist die Anamnese wichtig: die Erfassung der Schulbildung, der Berufsausbildung, der Berufsbewährung, der sozialen Integration und der Coping-Strategien bei früher durchgemachten Belastungen.

Die Motivation des Exploranden zur Wiederaufnahme einer Arbeit ist ein oft entscheidender
Faktor, kann aber weder gemessen noch direkt beobachtet werden. Nur Rückschlüsse aus der gesamten Situation von Umfeld und Befunden sind möglich, aber immer ein Stück weit spekulativ.

Ausserdem sind wir Ärzte Einzelkämpfer. Wir sind weitgehend auf die Angaben unseres Patienten/Exploranden angewiesen. Häufig könnte eine Kontaktnahme mit dem Arbeitgeber und anderen behandelnden Ärzten und betreuenden Instanzen weitere Aufschlüsse geben. Dies ist aber immer ein Aufwand, und zwar ein äusserer wie ein innerer. Es braucht das Einverständnis des Patienten. Es kann beim Patienten Misstrauen auslösen, und schliesslich besteht immer auch die Frage der Erreichbarkeit des Gesprächspartners. Wir Ärzte sind damit häufig der Suggestion unseres Patienten ausgesetzt. Als behandelnde Ärzte haben wir nicht nur Angst, den Patienten zu verlieren, wenn wir seine Arbeitsunfähigkeit hinterfragen oder relativieren. Wir haben auch Angst, dem Patienten Unrecht zu tun. Diese Angst ist heute verbreitet und findet sich bei allen beteiligten Personen. Die Angst, der Gemeinschaft der Prämienzahler Unrecht zu tun, ist bedeutend geringer. Dies könnte eine Spätfolge des rechten und linken Totalitarismus im 20. Jahrhundert sein, wo man den Individuen oft Unrecht getan hat zugunsten einer allgemeinen gesellschaftlichen Idee. Heute scheint das Pendel auf die Gegenseite ausgeschlagen zu haben.

Verbreitet ist auch das falsche Bild, dass mit dem Sprechen einer Rente dem Patienten geholfen werde. Untersuchungen belegen das Gegenteil [8]: Das Befinden der arbeitenden Patienten ist besser als dasjenige der Rentner. Die Berentung führt zur sozialen Isolation, zur Entwertung der Person und Chronifizierung der Gesundheitsstörungen. Obigem Irrtum erliegen oft auch Rechtsvertreter, die überzeugt sind, ihren Klienten etwas Gutes zu tun, wenn sie ihnen zu einer Rente verhelfen.

Bei den somatoformen Schmerzstörungen sind soziale Faktoren massgeblich für den Verlauf und die Prognose. Die Abklärung sollte möglichst früh und interdisziplinär stattfinden. Wenn ein solcher Patient ein Jahr lang oder länger rein somatisch untersucht und behandelt und anschliessend dem Psychiater überwiesen wird, ist der Fall längst chronifiziert und die Invalidisierung so weit fortgeschritten, dass der Prozess nicht mehr rückgängig zu machen ist.

Es könnte aus dem bisher Gesagten der falsche Eindruck entstanden sein, Patienten wollten grundsätzlich nicht arbeiten. Dies ist aber oft erst das Resultat einer ungünstigen Entwicklung. Zum Beispiel hoffen nach einem Unfall viele Patienten zunächst, den Einstieg ins Berufsleben wieder zu finden. Sie sind aber oft verloren und überfordert durch alle medizinischen, sozialen und rechtlichen Gegebenheiten. Mit zunehmendem zeitlichem Abstand zum Ereignis schwinden die Hoff-
Im Graubereich zwischen Körpe Psyche und sozialen Schwierigkeiten in Schweiz Med Wochenschr 1997;127:1430-9. 
nung und die Motivation, und es kommt die Resignation, falls der Patient nicht von einem Case Manager kompetent betreut und geleitet wird.

Die Frage der Arbeitsfähigkeit kann oft gar nicht rein aus medizinischer Sicht beantwortet werden. Es braucht Kenntnis der Tätigkeiten, der Verhältnisse am Arbeitsplatz sowie der vorherigen Bewährung des Patienten. Häufig ist ein Berufs- oder Rehabilitationsfachmann nötig.

Die Schwierigkeiten und der Aufwand einer seriösen Abklärung der Arbeitsfähigkeit sind der Gesellschaft bisher nicht bewusst gewesen. Ein sprechendes Beispiel dafür ist, dass bis zum April 2003 ein Arztzeugnis für die IV, welches oft ausschlaggebend für die Invalidisierung eines Patienten ist, mit Fr. 49.50 entschädigt worden ist. In diesen Zusammenhang gehört auch, dass die Gutachter häufig ein Feedback vermissen. Die Rechtsanwender scheinen sich nicht bewusst zu sein, dass Gutachter nicht im luftleeren Raum arbeiten können, sondern Informationen über die Weiterentwicklung ihrer Fälle brauchen, um ihre eigene Arbeit evaluieren und perfektionieren $\mathrm{zu}$ können. Die Gesellschaft idealisiert die Ärzte und meint, sie könnten alles einfach so. Wenn das dann nicht der Fall ist, ist die Gesellschaft enttäuscht.

Schliesslich sei noch erwähnt, dass in unserem System kaum Anreize zum Wiedereinstieg ins Erwerbsleben bestehen. Viele wollen lieber eine IV-Rente als Arbeitslosengelder beziehen [9]. Es besteht ein starker Trend, der als «einmal Rente immer Rente» bezeichnet werden kann. Auch hier gilt es, dass das System überdacht und Anreize geschaffen werden sollten.

Die Antwort auf die zweite Frage kann so zusammengefasst werden: Die Schwierigkeiten bei der Beurteilung der Arbeitsfähigkeit sind vielfältig. Von uns Ärzten wird etwas erwartet, wofür wir nicht ausgebildet sind, was anspruchsvoll und komplex ist und was wir oft aus rein medizinischer Sicht nicht beantworten können. Wenn die Gesellschaft von den Ärzten erwartet, dass weniger Menschen arbeitsunfähig erklärt und invalidisiert werden, ist das eine Überforderung der Ärzte. Alle Beteiligten müssen ihren Beitrag leisten, wenn dieses Ziel erreicht werden soll: die Politik, die Unternehmer, die Versicherungen und die Gerichte.

\section{Frage: \\ Wer muss welche Massnahmen treffen, um Verbesserungen bei der Beurteilung der Arbeitsfähigkeit zu ermöglichen?}

Die medizinischen Fakultäten sollten sich interdisziplinär intensiv mit dem Problem befassen, so dass sie den Ärzten in der Aus-, Weiter- und Fort- bildung ein besseres Instrumentarium vermitteln können. Es braucht die Fähigkeit, Risikopatienten zu erfassen, eine zumutbare Arbeitstätigkeit zu finden und verbesserte Rehabilitationsmassnahmen.

Wir behandelnde und begutachtende Ärzte sollten versuchen, das was wir können, besser zu tun. Zum Beispiel sollten der interdisziplinäre Dialog und der Kontakt mit dem Arbeitgeber vermehrt gesucht werden. Bei den Patienten sollten nicht nur die Einschränkungen, sondern vermehrt auch die vorhandenen Fähigkeiten erfasst und dargestellt werden. Andererseits sollten wir Ärzte das, was wir nicht können, deutlicher als bisher kommunizieren. Wir sollten die Idealisierung der Ärzte abbauen, sonst gibt es nur Enttäuschung und Schuldzuweisung.

Die Versicherungen sollten aktiver sein in der Erfassung von Risikopatienten sowie in der Evaluation der bisherigen Arbeitsbewährung und in der Fallführung allgemein. Bei Risikopatienten sollte ein aktives Case Management eingeführt werden, wie das z. B. die Suva vor kurzem begonnen hat.

Die Invalidenversicherung sollte die Bearbeitung ihrer Fälle beschleunigen. Sie sollte aktiver wieder eingliedern. Es sollte möglich sein, in den Regionalstellen der IV, welche neu gut dotiert sind mit arbeitsmedizinisch geschulten Ärzten und Berufsfachleuten, auf Zuweisung von behandelnden Ärzten und Versicherungen die Arbeitsfähigkeit in einem frühen Stadium interdisziplinär abzuklären. Falls dies aus gesetzlichen Gründen nicht möglich scheint, sollte es mindestens als Pilotprojekt in Angriff genommen werden. Ausserdem sollte die IV die Koordination mit der Unfallversicherung verbessern.

Die Unternehmer sollten vermehrt Arbeitsplätze schaffen für vermindert belastbare Personen.

Die Politik sollte das Versicherungssystem überdenken. Heute sind die Anreize für Renten gross und die Anreize zum Wiedereinstieg klein. Ausserdem sollte die Rechtsordnung überprüft werden.

Alle Beteiligten sollten ihr Menschenbild überdenken. Ist der Mensch nur Einflüssen von aussen ausgesetzt oder ist er eine Person, der eine Willensanstrengung zugemutet werden kann?

Die Gerichte sollten ihre Rechtsprechung überdenken. Diese Forderung mag vermessen erscheinen. Namhafte Vertreter der universitären Lehre in der Schweiz haben unmissverständlich geäussert, dass die Rechtsprechung im Bereich von Arbeitsfähigkeit und Invalidisierung nicht konsistent sei. 